Received: 24 May 2017

Accepted: 30 August 2017

Published online: 12 September 2017

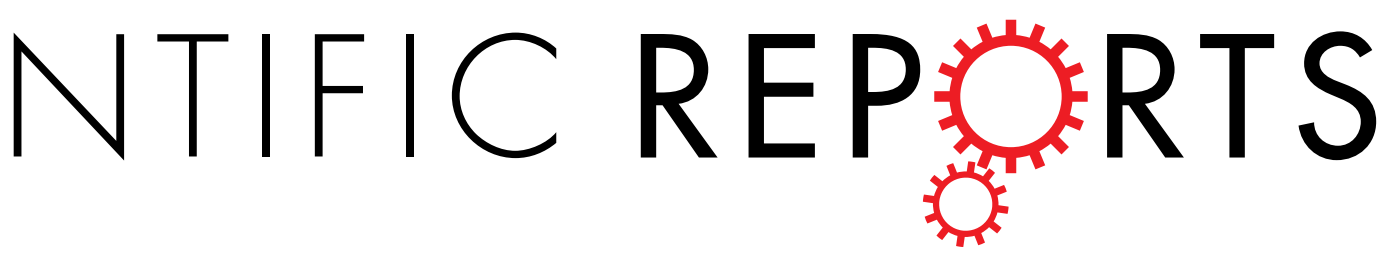

Investigating the neural correlates of smoking: Feasibility and results of combining electronic cigarettes

\section{with fMRI}

\author{
Matthew B. Wall $\mathbb{1}^{1,2,3}$, Alexander Mentink ${ }^{1,4}$, Georgina Lyons ${ }^{5}$, Oliwia S. Kowalczyk ${ }^{5,6}$, Lysia \\ Demetriou $^{1,2}$ \& Rexford D. Newbould ${ }^{1,2,7}$
}

Cigarette addiction is driven partly by the physiological effects of nicotine, but also by the distinctive sensory and behavioural aspects of smoking, and understanding the neural effects of such processes is vital. There are many practical difficulties associated with subjects smoking in the modern neuroscientific laboratory environment, however electronic cigarettes obviate many of these issues, and provide a close simulation of smoking tobacco cigarettes. We have examined the neural effects of 'smoking' electronic cigarettes with concurrent functional Magnetic Resonance Imaging (fMRI). The results demonstrate the feasibility of using these devices in the MRI environment, and show brain activation in a network of cortical (motor cortex, insula, cingulate, amygdala) and sub-cortical (putamen, thalamus, globus pallidus, cerebellum) regions. Concomitant relative deactivations were seen in the ventral striatum and orbitofrontal cortex. These results reveal the brain processes involved in (simulated) smoking for the first time, and validate a novel approach to the study of smoking, and addiction more generally.

Smoking is a major worldwide health problem, and current treatments for cigarette addiction are only partly effective. The behavioural and sensory aspects of smoking are thought to be important aspects of cigarette addiction, independently of the effects of nicotine ${ }^{1,2}$. Aspects of the multi-sensory (visual, tactile, olfactory, taste) experience of smoking can act as a powerful cue that reliably triggers craving and withdrawal symptoms in smoking addicts $^{3}$. This is consistent with the incentive-sensitization theory of addiction ${ }^{4,5}$, which proposes that neutral stimuli can promote drug-seeking behaviour through association with drug effects, and that this is mediated through sensitization of particular brain systems, principally the ventral striatum.

Research on the neural effects of smoking addiction has generally followed two largely independent paths. One has been concerned with the neurophysiological effects of nicotine and has generally used alternative routes of administration such as intravenous, cutaneous patch, or oral ${ }^{6-8}$. The second has used cigarette cues (usually images) to stimulate craving in (usually nicotine-abstinent) smokers, e.g. refs 9,10 . The former have provided usefully pure measures of the pharmacological effects of nicotine, and the latter have helped illuminate craving and drug-seeking mechanisms. However very few studies have investigated the neural effects of the most powerful cue associated with nicotine: the behavioural and multi-sensory repertoire of smoking itself. A small number of Positron Emission Tomography (PET) studies have involved subjects smoking while in a PET scanner. Berridge et al..$^{11}$ used radio-labelled ${ }^{[11 \mathrm{C}]}$ nicotine to investigate the pharmacokinetics of nicotine absorption, and Barrett et al. ${ }^{12}$ investigated the hedonic properties of smoking with the use of ${ }^{[11 \mathrm{C}]}$ raclopride to index dopamine release. The latter study showed that smoking-related changes in euphoria were related to dopamine release in the caudate

${ }^{1}$ Imanova Centre for Imaging Sciences, Burlington Danes Building, Hammersmith Hospital, Du Cane Road, London, W12 0NN, UK. 2Division of Brain Sciences, Imperial College London, Hammersmith Campus, Du Cane Road, London, UK. ${ }^{3}$ Clinical Psychopharmacology Unit, University College London, 1-19Torrington Place, London, WC1E 7HB, UK. ${ }^{4}$ Leiden University, Rapenburg 70, 2311 EZ, Leiden, The Netherlands. ${ }^{5}$ Department of Psychology, Royal Holloway University of London, Egham, Surrey, TW20 0EX, UK. ${ }^{6}$ Present address: Centre for Neuroimaging Sciences, Institute of Psychiatry, Psychology and Neuroscience, Kings College London, London, UK. ${ }^{7}$ Present address: Perspectum Diagnostics, Oxford, UK. Correspondence and requests for materials should be addressed to M.B.W. (email: matt. wall@imanova.co.uk) 

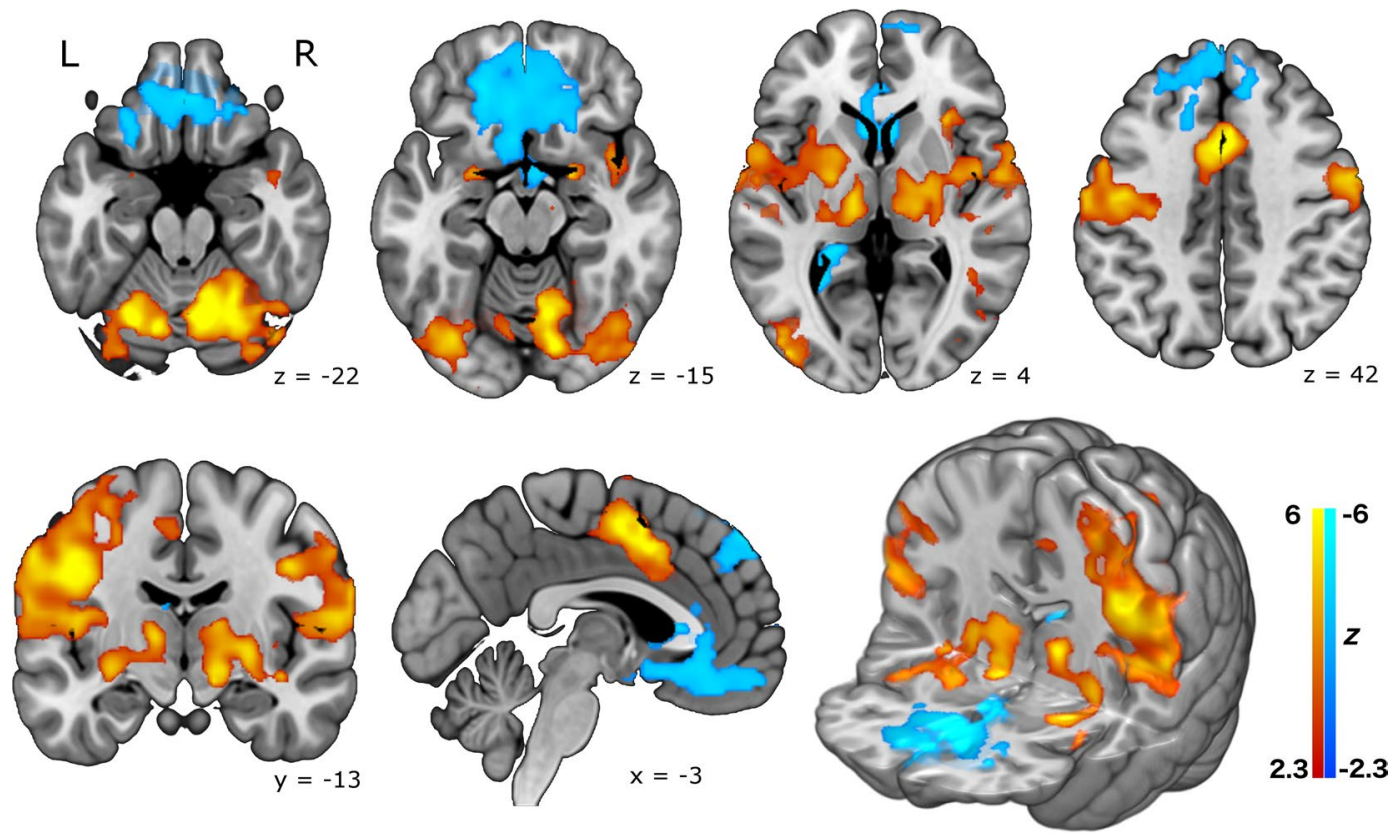

Figure 1. Brain activation in the cued smoking experiment, from a group mean analysis of 10 healthy smokers, including physiological noise modelling. Top row: axial slices. Bottom row: (from left) coronal slice, sagittal slice, and a 3D rendered image. Increased activity (red-yellow) is seen in the amygdala, cerebellum, thalamus, putamen, globus pallidus, insula, cingulate gyrus and motor cortex. Reduced activity (blue-cyan) is seen in the ventral striatum, orbitofrontal cortex, and dorso-medial frontal regions. Statistical maps are shown thresholded at $Z>2.3, p<0.05$ (cluster corrected for multiple comparisons) and neurological convention is used $(\mathrm{L}=\mathrm{Left}$, $\mathrm{R}=$ Right). Background anatomical image is a high-resolution version of the MNI152 T1 anatomical template.

and putamen, though not in the ventral striatum. Domino et al. ${ }^{13}$ used regular and denicotinized cigarettes to examine dopamine release; both showed effects on dopamine throughout the striatum, but the denicotinized cigarettes led to significantly less dopamine release. Cosgrove et al. ${ }^{14}$ also identified a potential sex difference in striatal dopamine release, with male subjects showing a more consistent and rapid response to smoking.

These PET studies are restricted, by the nature of the method, to examining a single neurotransmitter system. To date, there have been no investigations of active smoking using a more general neuroimaging method which could reveal effects across the entire brain, such as functional Magnetic Resonance Imaging (fMRI). This is likely because a host of practical, health, and safety issues largely preclude the use of traditional (i.e. combustible tobacco) cigarettes in the modern neuroscientific laboratory environment. For example: most MRI scanners are enclosed and restrictive, air capture/filtration systems would be required to deal with the smoke, and the procedure may require a naked flame. In addition local or national regulations may prohibit smoking within research institutions or hospital sites (e.g. Health Act 2006, in the United Kingdom). One group has published two technical papers ${ }^{15,16}$ describing an MRI compatible ventilation system to enable smoking during functional imaging, however they have never presented any fMRI data collected using the device. Another recent paper ${ }^{17}$ describes an MR-compatible olfactometer designed to deliver the odour of a burning cigarette to participants. Both these accounts use specialist equipment to mitigate some of the practical difficulties of smoking within an MRI scanner.

Many of these issues may be entirely obviated by the use of electronic cigarettes, or more accurately, Electronic Nicotine Delivery Systems $\left(\right.$ ENDS $\left.^{18}\right)$. These are relatively novel consumer products that deliver nicotine, and are designed to provide a closer simulation of smoking tobacco cigarettes than previous nicotine-containing products. Use of ENDS does not involve combustion, and most produce relatively small amounts of vapour that evaporates within a few seconds. Evidence suggests that ENDS are effective at reducing withdrawal symptoms in nicotine-deprived smokers ${ }^{19}$ and preliminary data show that ENDS may be effective at helping smokers quit, or reducing their smoking ${ }^{20,21}$, although see ${ }^{22}$ for a contrasting view. As such, they may hold great potential as a replacement for traditional cigarettes, and be a major benefit to public health ${ }^{18}$, but as a novel technology, their effects on the brain, general health, and patterns of tobacco use is still largely unknown.

We have explored the use of ENDS in combination with functional Magnetic Resonance Imaging (fMRI) in order to visualise brain activity related to active smoking, or more strictly, to the close simulation of active cigarette smoking that ENDS provide. To achieve this we first undertook testing of commercially available ENDS to assess the devices for magnetic susceptibility and potential effects on MRI image quality. After successfully identifying a device that had minimal magnetic susceptibility and no discernible effects on image quality, 11 healthy smokers completed a scanning session that included active smoking fMRI tasks. In the first task subjects were instructed to 'smoke' (i.e. inhale on the ENDS) by visual cues. In the second scan subjects received no cues, and were instructed to 'smoke' naturally, ad libitum. 

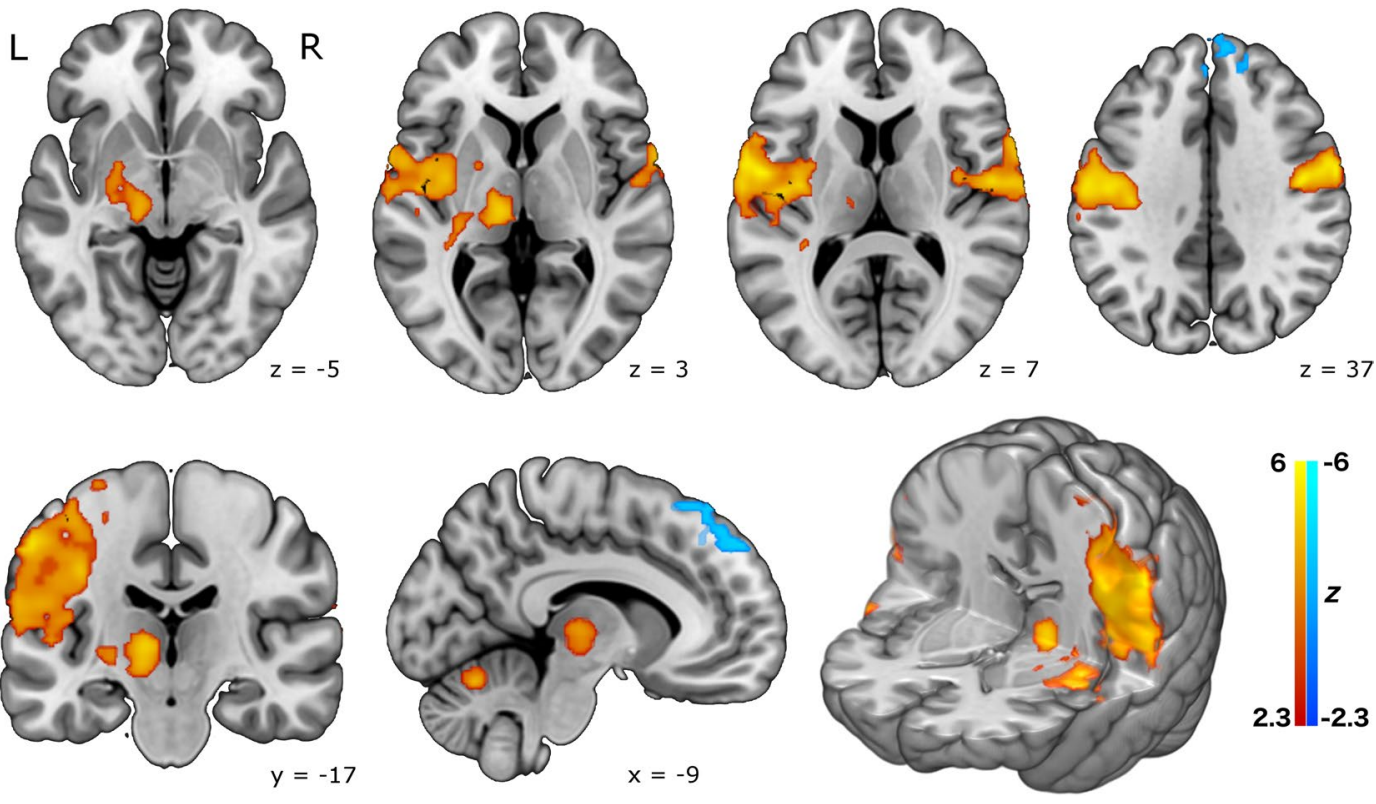

Figure 2. Brain activation in the naturalistic smoking experiment, from a group mean analysis of 10 healthy smokers, including physiological noise modelling. Top row: axial slices. Bottom row: (from left) coronal slice, sagittal slice, and a 3D rendered image. The pattern of activation is broadly similar to the cued experiment, but somewhat less robust, and less widespread. Statistical maps are shown thresholded at $Z>2.3, p<0.05$ (cluster corrected for multiple comparisons) and neurological convention is used ( $\mathrm{L}=\mathrm{Left}, \mathrm{R}=\mathrm{Right})$. Background anatomical image is a high-resolution version of the MNI152 T1 anatomical template.

\section{Results}

ENDS MR compatibility. None of the five ENDS contained ferromagnetic components such as might experience torque inside the magnetic field, however, two were found to have metallic bodies upon disassembly (see Table 2). While no large torques would be expected, eddy currents will be induced in the conductive body from the MRI's RF activity ${ }^{23}$. These eddy currents generate unwanted counter-acting magnetic fields, and may pose a safety risk from heating of the conductor. Further, most metals have a magnetic susceptibility, $\chi$, far enough $\left(>10^{-5}\right)$ from the $\chi$ of water that image distortion is expected. While an aluminium body would have a modest effect, nickel or stainless steel would create large image distortions. If the ENDS could be used parallel to the main magnetic field B0, the cylinder-shaped body would not perturb B0 outside the ENDS. However, when used normally the ENDS would be transverse to the main magnetic field; resulting in a dipole perturbation in cylindrical coordinates $(\rho, \phi)$ of $\Delta B_{Z}=\frac{\Delta \chi B_{0}}{8 \rho^{2}}\left(2 \cos ^{2} \varphi-1\right)$. These characteristic dipole patterns are illustrated in Supplementary Figure 1.

The MRI environment had no apparent effect on the operation of any of the tested ENDS devices. The magnetic susceptibility artefacts produced by the different devices varied widely (see Supplementary Figure 2 for high and low-susceptibility examples, with artefacts produced by the eddy currents mentioned above). Unsurprisingly, the larger devices with metal construction produced the most detrimental effects, but even the smaller devices produced some areas of signal loss on the MRI phantom images. However, the smaller devices produced no obvious image artefacts in the test with a human subject, suggesting that they would be suitable for use in the main experiment.

Task Results. In the main study, Fig. 1 illustrates the resulting pattern of brain activity that occurred in the cued-smoking task, time-locked to the smoking events. Large activation clusters are seen in the dorsal motor cortex in the left hemisphere, consistent with movements of the right hand during smoking trials. More lateral motor activity likely represents oro-facial movements on smoking trials. Other cortical regions strongly activated were the mid-insula, the amygdala, and the (dorsal) anterior cingulate gyrus. Strong activation of sub-cortical regions is also seen, principally in the thalamus, globus pallidus, and putamen. Relative deactivations associated with smoking events were also seen in a large region encompassing parts of the ventral striatum (nucleus accumbens and ventral caudate) and extending forward into orbitofrontal cortex. An additional area of deactivation was also seen in more dorsal frontal cortex.

Brain activity in the naturalistic smoking experiment showed a similar pattern, though less strongly, and less widespread, with significant clusters in motor cortex, thalamus, globus pallidus and putamen during smoking events. Relative deactivations were restricted to the dorsal frontal region in this analysis (see Fig. 2).

Additional analyses of data from both tasks that did not include the physiological noise modelling regressors produced a highly similar set of results (see Supplementary Figure 3), suggesting that physiological effects are not a significant confound in the data. Table 1 lists location of each activation cluster in all analyses, in MNI coordinate space. 


\begin{tabular}{|c|c|c|c|}
\hline Task/Contrast & Region & Right Hemisphere & Left Hemisphere \\
\hline \multirow{10}{*}{ Cued smoking (activations) } & Cerebellum VI & $24-60-26$ & $-26-64-24$ \\
\hline & Amygdala & $220-16$ & $-240-16$ \\
\hline & Insula & $408-10$ & $-386-6$ \\
\hline & Putamen & $240-6$ & $-28-4-6$ \\
\hline & Pallidum & $24-104$ & $-24-60$ \\
\hline & Thalamus & $14-142$ & $-12-202$ \\
\hline & Precentral gyrus (dorsal) & $50-452$ & $-46-652$ \\
\hline & Precentral gyrus (ventral) & $54-426$ & $-50-826$ \\
\hline & Cingulate gyrus & 21242 & -21042 \\
\hline & Fusiform gyrus & $40-72-14$ & $-36-76-14$ \\
\hline \multirow{4}{*}{ Cued smoking (deactivations) } & Medial frontal cortex & $638-16$ & $-1034-12$ \\
\hline & Nucleus accumbens & $410-10$ & $-88-12$ \\
\hline & Caudate & 8186 & -8166 \\
\hline & Frontal pole & 144830 & -144828 \\
\hline \multirow{7}{*}{ Naturalistic smoking (activations) } & Cerebellum VI & $16-64-22$ & $-16-66-20$ \\
\hline & Insula & - & $-40-40$ \\
\hline & Putamen & - & -2442 \\
\hline & Pallidum & - & $-24-6-4$ \\
\hline & Thalamus & - & $-12-200$ \\
\hline & Precentral gyrus (dorsal) & $52-442$ & $-50-1242$ \\
\hline & Precentral gyrus (ventral) & 60022 & $-56-422$ \\
\hline Naturalistic smoking (deactivations) & Frontal pole & 163846 & -64844 \\
\hline
\end{tabular}

Table 1. Coordinates of the approximate centre of activation clusters within anatomical regions for all experiments and contrasts. Coordinates are in MNI space.

\section{Discussion}

These data demonstrate the feasibility of using ENDS in the MRI environment, and reveal the neural correlates of (simulated) smoking for the first time. A wide network of activated areas included cortical (motor cortex, insula, cingulate, amygdala) and sub-cortical (thalamus, putamen, globus pallidus, cerebellum) regions, with corresponding significant deactivations in ventral striatum and orbitofrontal cortex.

The most prominent result is the activation in left motor cortex which can plausibly be attributed to hand and oro-facial movements associated with the smoking task. The activation clusters in the right cerebellum are also most plausibly related to the motor components of the task. While certainly part of the behavioural repertoire of smoking, these results are unsurprising, and will not be discussed further. Of more interest are the results in other regions, particularly the thalamus and striatum. Previous work has identified differences in dopamine release in the caudate and putamen in smokers vs. non-smokers ${ }^{24}$, and the striatum is widely considered to be a key set of brain structures that support addiction ${ }^{25-27}$, with interactions between nicotine and the mesolimbic dopamine system also well-established (for a review $\mathrm{se}^{28}$ ). Intriguingly, parts of the ventral striatum (nucleus accumbens, and ventral caudate) appear to show a relative signal decrease in response to smoking in this experiment, as well as a large region in the orbitofrontal cortex. One possible explanation for these regions of relative deactivation may be the shift from ventral to dorsal striatum that occurs as drug use becomes habitual and compulsive $e^{29}$, and the finding that dysfunctional inhibitory mechanisms in the prefrontal cortex also play a role in addictive behaviour ${ }^{29,30}$. Janes et al. ${ }^{31}$ also recently showed that the connectivity of the orbitofrontal cortex was highly related to subjective craving measures. The current findings are consistent with the established role of the ventral striatum and frontal cortex in drug craving, and further suggest that activity in these areas is actually reduced during active drug consumption, with the rewarding aspect of consumption mediated by more dorsal striatal regions. The results are also highly consistent with the previous PET study by Berridge et al. ${ }^{11}$ which showed smoking-related euphoria was related to dopamine release in the dorsal, but not ventral, striatum. The general activation pattern involving the brain's reward circuitry also implies that use of ENDS may have a similar rewarding effect as traditional cigarettes.

It is important to note that the current data say relatively little about the neural effects of nicotine. Although the ENDS used contained nicotine, the modelling of the brain response was time-locked to the action of smoking. Nicotine (absorbed into the blood through the vapour produced by the ENDS) is likely to only enter the brain between 5 and 20 seconds after each inhalation ${ }^{11}$, and modelling this effect within a conventional fMRI design would be difficult, since the timing of the nicotine 'hit' after each trial is somewhat uncertain. There is a large literature on nicotine and its pure pharmacological effects are relatively well understood ${ }^{6,7}$. The current data stand as complementary to this literature, and provide a visualisation of brain processes related to the consumption of nicotine in a relatively naturalistic manner. Future studies which seek to improve on this methodology may concentrate more on the effects of nicotine consumed in this way, and use nicotine deprived subjects, with pre- and post-scan measures of urine or plasma nicotine concentrations.

Results from the cued task were robust, while results from the naturalistic task were noticeably muted in comparison. There are several plausible explanations for this finding. Firstly, in the naturalistic study the number 
of smoking events varied across subjects $($ mean $=28.8, \mathrm{SD}=15.4)$ and this produced more between-subject variance in the data. Secondly, the timing of events in the naturalistic study was determined by the subjects, and therefore did not necessarily conform to optimal principles of fMRI experimental design ${ }^{32,33}$; this could affect the signal-detection power of the experiment. Thirdly, the naturalistic scan was always conducted after the cued scan and subjects were possibly nicotine-sated in the latter scan, leading to less activation in the striatal (i.e. more reward-related) regions. Fourth, the task demands of each scan were quite different, with one requiring active focus and attention on the external cue task, while the other did not. One other aspect of the design deserves comment, which is the use of a purely baseline control condition in both tasks. In this initial study we were concerned with demonstrating the technical feasibility of using ENDS in this way, but also with visualising the neural correlates of the entire behavioural and sensory repertoire of smoking. The comparison of 'smoking' with 'not smoking' (that is, a resting baseline) provides this. Future work may be concerned with dissecting the various components of the response using appropriate control conditions. For instance, sham-smoking with a non-functional device may be a useful control condition for subtracting out the motor aspects of the response.

Future work using similar methods may also be focussed on optimising the equipment, procedure, and analysis strategy. The market for electronic cigarettes has shown explosive growth in recent years, and new products are being launched almost daily. The first generation 'cig-a-like' devices used here are designed to mimic traditional cigarettes, and this visual mimicry may be helpful in reducing withdrawal symptoms ${ }^{34}$. However the second or third generation devices currently on the market appear to be more effective at delivering nicotine, and thereby reducing craving and withdrawal symptoms $\mathrm{s}^{34,35}$. Unfortunately these devices tend to be larger, heavier, and incorporate more metallic components, making them unsuitable for use in the MRI environment without substantial modification. The smaller, first generation devices (particularly those that have a plastic construction and use non-magnetic Lithium polymer or 'LiPo' batteries) are probably the best current option for this kind of work. The other useful feature of these devices is the end-mounted LED, which allows for easy monitoring of task compliance, with the simple device used here. Alternative analysis strategies for studies of this type may be focussed on visualising the trial-by-trial effect of nicotine on the brain, or the cumulative effect over the course of the experiment. This would be difficult because of the reasons mentioned above, but would perhaps be feasible using a more flexible statistical model, or model-free analysis approaches such as Independent Components Analysis (ICA).

It is important to note that, while ENDS provide the closest simulation of smoking tobacco cigarettes currently available, the experience of smoking is qualitatively somewhat different to that of 'vaping' using ENDS. Smoking produces strong characteristic flavours, odours, and a harsh 'throat hit', all of which (while present) are noticeably muted in the small, relatively low-power ENDS used here. In addition, while ENDS manufacturers often try to approximate the flavour of burning tobacco, the actual flavour of tobacco cigarettes may also be qualitatively different. The relative deactivations observed in orbitofrontal cortex (generally recognised as an area that responds to olfactory stimuli ${ }^{36}$ ) may therefore be a result of the less powerful olfactory properties of ENDS, and this area (plus other olfactory areas such as the piriform cortex) may respond more strongly to tobacco cigarette smoking. Other subjective sensations related to tobacco smoking (such as the strong 'throat hit', or the effect of nicotine on the mucosal membranes) may also be somewhat inadequately represented by the current data. However, previous work ${ }^{19}$ has also shown that ENDS are highly effective in reducing craving and withdrawal symptoms, even in previously ENDS-naïe smokers. This suggests that despite the somewhat different subjective sensations, ENDS provide a 'good enough' simulation of smoking to substitute for cigarettes, and can therefore produce similarly rewarding effects to cigarettes (e.g. reductions in craving), in cigarette-addicted subjects.

This demonstration of the feasibility of using ENDS in the MRI environment has served to validate an entirely novel approach to the study of cigarette dependence, and the more general brain mechanisms of addiction. We have also revealed for the first time the full neural effects of active (simulated) smoking, which includes activation in a network of cortical (motor, insula, cingulate, amygdala) and sub-cortical (putamen, thalamus) regions, with relative deactivation in ventral striatum and orbitofrontal cortex. Together with previous work on nicotine, and cue-reactivity in smokers, these findings provide a more complete picture of the neural effects associated with cigarette smoking, and addiction in general.

\section{Materials and Methods}

Equipment. The ENDS devices were all widely commercially available at the time of testing (July, 2014). The five brands tested were: 'Njoy', 'Puritane', 'Vype', 'Nucig', and 'Jasper \& Jasper.' See Table 2 for more details on each device. These were all first generation, or 'cig-a-like' devices, with a form factor designed to mimic traditional cigarettes. All were of similar construction, consisting of an outer body, a lithium-polymer (LiPo) battery, a pressure-activated LED, a resistive heating element and nicotine-soaked wadding. LiPo batteries are commonly used in devices designed for the MRI environment, as they contain no internal metal components. All were operated by inhalation, and all incorporated a LED at the end of the device, intended to mimic the burning ember of a traditional cigarette. We exploited this feature by using a custom-built opto-electronic device to record the output of the LED at the end of the ENDS (see supplementary methods for details). This enabled effective and easy monitoring of task compliance, and recorded a time-series to be used in the analysis of the naturalistic smoking task (see below).

Initial Product Testing. We tested five different brands of commercially available ENDS for their suitability for use in the MRI environment; Table 2 lists their basic characteristics. None were modified or tampered with in any manner. All five ENDS were immersed in a $2 \%$ agarose solution with $160 \mathrm{mM} \mathrm{NaCl}$ for conductivity. This phantom was imaged on a Siemens $3 \mathrm{~T}$ Verio MRI scanner (Siemens Healthcare, Erlangen Germany). Imaging consisted of a gradient-echo sequence; TR $=300 \mathrm{~ms}$, TE $1=5.19 \mathrm{~ms}$, TE $2=7.65 \mathrm{~ms}$, flip angle $=60^{\circ}, 1.5 \times 1.5 \times 2$ $\mathrm{mm}$ voxels, 35 axial slices, bandwidth $=1520 \mathrm{~Hz} /$ pixel at two echo times: 1.93 and $4.39 \mathrm{~ms}$. A phase difference image between the two echo times was used to characterize the disturbance to the magnetic field (B0) from 


\begin{tabular}{|l|l|l|l|l|l|l|}
\hline Brand & Variety/model & $\begin{array}{l}\text { Manufacturers } \\
\text { stated nicotine } \\
\text { content }\end{array}$ & $\begin{array}{l}\text { Manufacturers stated } \\
\text { flavouring }\end{array}$ & Body construction & $\begin{array}{l}\text { Length } \\
(\mathbf{m m})\end{array}$ & Battery \\
\hline Njoy & Gold & $3 \%$ (by weight) & 'Gold tobacco' & Plastic & 84 & LiPo, 70mAh \\
\hline Puritane & Disposable & $16 \mathrm{mg} / \mathrm{g}$ & 'Original flavour' & Metallic & 108 & LiPo, 240mAh \\
\hline Vype & Bold & $18.6 \mathrm{mg}$ & 'Classic flavour: Bold' & Plastic & 85 & LiPo, 280mAh \\
\hline Nucig & N/A & $18 \mathrm{mg}$ & 'Tobacco' & Metallic & 106 & LiPo, 200mAh \\
\hline $\begin{array}{l}\text { Jasper \& } \\
\text { Jasper }\end{array}$ & Nano disposable & $16 \mathrm{mg}$ & 'Tobacco flavour' & $\begin{array}{l}\text { Plastic; metal } \\
\text { housing around } \\
\text { heating element }\end{array}$ & 88 & LiPo, 170mAh \\
\hline
\end{tabular}

Table 2. Characteristics of the ENDS devices used in the initial testing phase.

each ENDS. A spin-echo sequence was used to characterize disturbances to the applied RF (B1) field; $T R=5 \mathrm{~s}$, $\mathrm{TE}=8.5 \mathrm{~ms}, 1.2 \times 1.2 \times 3.5 \mathrm{~mm}$ voxels, 40 axial and coronal slices, bandwidth $=800 \mathrm{~Hz} / \mathrm{pixel}$.

Subsequently, one subject (Author MBW) completed a scan using each of the five brands in a similar manner to the subjects in the main experiment (described below). These scans used a dual-echo, echo-planar imaging (EPI) sequence for BOLD contrast with 36 axial slices, aligned with the AC-PC axis (TR $=2000 \mathrm{~ms}$, TE $1=13 \mathrm{~ms}$, TE2 $=31 \mathrm{~ms}$ flip angle $=80^{\circ}, 3 \mathrm{~mm}$ isotropic voxels, parallel imaging factor $=2$, bandwidth $=2298 \mathrm{~Hz} /$ pixel). The ENDS devices were therefore assessed for general magnetic susceptibility, and in particular for their potential to generate artefacts when used by a subject during a BOLD EPI acquisition.

Subjects. Subjects were 11 (3 females) daily or semi-regular social smokers, who were in good general health. One (male) subject was subsequently excluded from analysis because of excessive ( $>5 \mathrm{~mm}$ ) head movement and failure to comply with the task, which left a final group of 10 (mean age of 29.1 years; SD =5.91). The mean number of cigarettes smoked daily for this group was $10.1(\mathrm{SD}=5.6)$. Subjects were not asked to abstain from smoking on the day of the scan, and were therefore not in a nicotine-deprived state. All subjects had some previous familiarity with ENDS, but none were regular users of ENDS. Informed consent was obtained from all subjects, and all procedures were conducted in accordance with the Declaration of Helsinki (seventh revision, 2013). All scans were performed under local institution-approved guidelines for fMRI scans on healthy subjects to ensure adherence to ICH-GCP standards.

Tasks and scanning procedure. Data were acquired on a Siemens $3 \mathrm{~T}$ Magnetom Trio MRI scanner (Siemens Healthcare, Erlangen, Germany), equipped with a 32-channel phased-array head coil. Subjects held the ENDS in their right-hand, with the right elbow cushioned so that they could comfortably hold it on their chest, close to their mouth, in order to minimise the hand movement required on each trial. Subjects could view a back-projected image on a screen in the rear of the scanner bore via a mirror mounted on the head-coil. They were instructed to try to avoid looking down (e.g. along the scanner bore, towards the ENDS) as they inhaled, to counteract the natural tendency to nod the head forward slightly when looking down.

Based on the results of the initial testing (see results section) the 'Njoy' device was selected as a good candidate device for use in the main experiment. A fresh ENDS was used for each subject, and the only modification made was the removal of the plastic end cap covering the LED. This was required to provide higher light output and a better signal for the opto-electronic recording device. The optical fibre was attached to the end of the ENDS by a rubber connector, and remained in place throughout the scan. The output of the device was recorded by a standard analogue-to-digital recording system (PowerLab 8/35, AD Instruments, Oxford UK), and physiological parameters (respiration via a respiratory belt around the subjects' chest, and cardiac data via a pulse-oximeter on the index finger of the left hand) were recorded on the same system.

At the beginning of the scan session high-resolution T1-weighted anatomical images were acquired using a magnetization prepared rapid gradient echo (MPRAGE) sequence with parameters from the Alzheimer's Disease Research Network (ADNI; 160 slices $\times 240 \times 256, \mathrm{TR}=2300 \mathrm{~ms}, \mathrm{TE}=2.98 \mathrm{~ms}$, flip angle $=9^{\circ}, 1 \mathrm{~mm}$ isotropic voxels, bandwidth $=240 \mathrm{~Hz} /$ pixel, parallel imaging factor $=2{ }^{37}$ ) along with B0 field-map images (sequence as described above). Subjects then completed two functional scans (sequence as described above) of ten minutes duration each. The first was the cued smoking task that consisted of 20 trials with inter-trial intervals that varied randomly between 20,25 , and 30 seconds (mean $=25 \mathrm{~s}$ ). On each trial, a three second countdown $(3,2,1)$ was displayed in the centre of the screen, followed by the word 'SMOKE', displayed for two seconds. A fixation cross was present throughout the inter-trial interval. Participants were instructed to time their inhalations on the ENDS to coincide with the 'SMOKE' cue. The second task was the naturalistic smoking task, where there were no visual cues, and subjects were instructed to smoke 'naturally' throughout the ten minute scan.

Data analysis. All analysis was conducted using FSL ${ }^{38,39}$ version 5.0. Pre-processing of the functional data involved removal of non-brain tissue, head-motion correction, spatial smoothing with a $6 \mathrm{~mm}$ full-width-half-maximum Gaussian kernel, and high-pass temporal filtering with a cut-off of $100 \mathrm{~s}$. Additional correction for the effects of head-motion used the ICA-AROMA tool (ICA-based Automatic Removal Of Motion Artefacts; ${ }^{40,41}$ ). For the cued smoking task data, first-level statistical (General Linear Model) models were created which contained a single regressor of interest. This time-series modelled two-second events defined by the occurrence of the smoking cue, convolved with a gamma function to produce a standard model of the haemodynamic response function. The first temporal derivative of this time-series was also included in the model. Statistical 
maps resulting from these analyses were coregistered to each subjects' skull-stripped T1 anatomical image, and then to an image in standard stereotactic space (the MNI152 template provided with FSL). Analysis at the second (group) level computed a simple mean across subjects for the regressor of interest using FSL's FLAME-1 model, and the results were thresholded at $Z=2.3$ ( $p<0.05$, cluster-corrected for multiple comparisons).

For the naturalistic smoking task data, 'smoking' events were defined based on the recorded output of the opto-electronic device. This enabled a custom regressor of smoking events to be produced for each subject, using custom written MatLab (MathWorks Ltd.) code. The temporal derivative of the raw time-series was computed, and the peaks and troughs of the derivative time-series were used to define the start and end of each smoking event. In all other respects analysis of this task was identical to the cued smoking task.

Because of the established effects of physiological parameters on fMRI data (e.g. refs 42-44), and because these tasks crucially depend on timed inhalations, analysis models of both sets of data also included physiological noise regressors. The recorded physiological (cardiac and respiratory) data was processed using the Physiological Noise Modelling (PNM) toolbox included with $\mathrm{FSL}^{45}$ and an additional 12 Fourier-expanded regressors were created to model cardiac and respiratory function. Additional analyses were conducted without the physiological noise regressors, to examine the effect of the noise modelling procedure.

Data availability. Unthresholded statistical maps resulting from the group-level analyses (both physiologically-corrected and standard versions) are available on the NeuroVault repository (http://neurovault. org/collections/2495/). All raw data, stimulus code, and analysis code are available from the corresponding author on reasonable request.

\section{References}

1. Rose, J. E. Nicotine and nonnicotine factors in cigarette addiction. Psychopharmacology (Berl). 184, 274-85 (2006).

2. Balfour, D. The neurobiology of tobacco dependence: A preclinical perspective on the role of the dopamine projections to the nucleus. Nicotine Tob. Res. 6, 899-912 (2004).

3. Niaura, R. et al. Individual differences in cue reactivity among smokers trying to quit: effects of gender and cue type. Addict. Behav. 23, 209-224 (1998).

4. Robinson, T. \& Berridge, K. The neural basis of drug craving: an incentive-sensitization theory of addiction. Brain Res. Rev. 8 , 247-291 (1993)

5. Robinson, T. E. \& Berridge, K. C. Incentive-sensitization and addiction. Addiction 96, 103-14 (2001).

6. Stein, Ea et al. Nicotine-induced limbic cortical activation in the human brain: a functional MRI study. Am. J. Psychiatry 155, 1009-15 (1998)

7. Yamamoto, R. T. et al. Nicotine related brain activity: The influence of smoking history and blood nicotine levels, an exploratory study. Drug Alcohol Depend. 129, 137-144 (2013).

8. Cole, D. M. et al. Nicotine replacement in abstinent smokers improves cognitive withdrawal symptoms with modulation of resting brain network dynamics. Neuroimage 52, 590-9 (2010).

9. David, S. P. et al. Ventral striatum/nucleus accumbens activation to smoking-related pictorial cues in smokers and nonsmokers: a functional magnetic resonance imaging study. Biol. Psychiatry 58, 488-94 (2005).

10. Janes, A. C. et al. Memory retrieval of smoking-related images induce greater insula activation as revealed by an fMRI-based delayed matching to sample task. Addict. Biol. doi:https://doi.org/10.1111/adb.12112 (2013).

11. Berridge, M. S. et al. Smoking produces rapid rise of [11C]nicotine in human brain. Psychopharmacology (Berl). 209, 383-94 (2010).

12. Barrett, S. P., Boileau, I., Okker, J., Pihl, R. O. \& Dagher, A. The hedonic response to cigarette smoking is proportional to dopamine release in the human striatum as measured by positron emission tomography and [11C]raclopride. Synapse 54, 65-71 (2004).

13. Domino, E. F. et al. Denicotinized versus average nicotine tobacco cigarette smoking differentially releases striatal dopamine. Nicotine Tob. Res. 15, 11-21 (2013).

14. Cosgrove, K. P. et al. Sex Differences in the Brain's Dopamine Signature of Cigarette Smoking. J. Neurosci. 34, 16851-5 (2014).

15. Frederick, B. \& Lindsey, K. An MR-compatible device for delivering smoked marijuana during functional imaging. Pharmacol. ... 87, 81-89 (2007).

16. Lindsey, K. P. et al. Design and validation of an improved nonferrous smoking device for self-administration of smoked drugs with concurrent fMRI neuroimaging. Clin. EEG Neurosci. 40, 21-30 (2009).

17. Lowen, S. B., Farmer, S. L. \& Lukas, S. E. Improved low-cost, MR-compatible olfactometer to deliver tobacco smoke odor. Behav. Res. Methods 49, 74-82 (2017).

18. Nutt, D. J. et al. Estimating the Harms of Nicotine-Containing Products Using the MCDA Approach. Eur. Addict. Res. 20, 218-225 (2014).

19. Dawkins, L., Turner, J., Hasna, S. \& Soar, K. The electronic-cigarette: effects on desire to smoke, withdrawal symptoms and cognition. Addict. Behav. doi:https://doi.org/10.1016/j.addbeh.2012.03.004 (2012).

20. Rahman, M. A., Hann, N., Wilson, A., Mnatzaganian, G. \& Worrall-Carter, L. E-Cigarettes and smoking cessation: Evidence from a systematic review and meta-analysis. PLoS One 10, 1-16 (2015).

21. Bullen, C. et al. Electronic cigarettes for smoking cessation: a randomised controlled trial. 6736, 1-9 (2013).

22. Kalkhoran, S. \& Glantz, S. A. E-cigarettes and smoking cessation in real-world and clinical settings: A systematic review and metaanalysis. Lancet Respir. Med. 4, 116-128 (2016).

23. Schenck, J. F. The role of magnetic susceptibility in magnetic resonance imaging: MRI magnetic compatibility of the first and second kinds. Med. Phys. 23, 815-850 (1996).

24. Takahashi, H. et al. Enhanced dopamine release by nicotine in cigarette smokers: a double-blind, randomized, placebo-controlled pilot study. Int. J. Neuropsychopharmacol. 11, 413-7 (2008).

25. Volkow, N. D., Fowler, J. S., Wang, G.-J., Swanson, J. M. \& Telang, F. Dopamine in Drug Abuse and Addiction. Arch. Neurol. 64, 1575-1579 (2007).

26. Robbins, T. \& Everitt, B. Limbic-Striatal Memory Systems and Drug Addiction. Neurobiol. Learn. Mem. 78, 625-636 (2002).

27. Everitt, B. J. \& Robbins, T. W. Neural systems of reinforcement for drug addiction: from actions to habits to compulsion. Nat. Neurosci. 8, 1481-1489 (2005).

28. Pierce, R. C. \& Kumaresan, V. The mesolimbic dopamine system: The final common pathway for the reinforcing effect of drugs of abuse? Neurosci. Biobehav. Rev. 30, 215-238 (2006)

29. Everitt, B. J. \& Robbins, T. W. From the ventral to the dorsal striatum: Devolving views of their roles in drug addiction. Neurosci. Biobehav. Rev. 37, 1946-1954 (2013).

30. Goldstein, R. Z. \& Volkow, N. D. Dysfunction of the prefrontal cortex in addiction: neuroimaging findings and clinical implications. Nat. Rev. Neurosci. 12, 652-669 (2012). 
31. Janes, A. C., Farmer, S., Frederick, B. D., Nickerson, L. D. \& Lukas, S. E. An increase in tobacco craving is associated with enhanced medial prefrontal cortex network coupling. PLoS One 9, 1-5 (2014).

32. Friston, K. J., Zarahn, E., Josephs, O. \& Henson, R. N. \& Dale, a. M. Stochastic designs in event-related fMRI. Neuroimage 10, 607-19 (1999).

33. Dale, a. M. Optimal experimental design for event-related fMRI. Hum. Brain Mapp. 8, 109-14 (1999).

34. Dawkins, L., Kimber, C., Puwanesarasa, Y. \& Soar, K. First- versus second-generation electronic cigarettes: predictors of choice and effects on urge to smoke and withdrawal symptoms. Addiction 110, 669-677 (2015).

35. Lechner, W. V. et al. The comparative efficacy of first- versus second-generation electronic cigarettes in reducing symptoms of nicotine withdrawal. Addiction 110, 862-867 (2015).

36. Gottfried, J. a. \& Zald, D. H. On the scent of human olfactory orbitofrontal cortex: meta-analysis and comparison to non-human primates. Brain Res. Brain Res. Rev. 50, 287-304 (2005).

37. Jack, C. R. et al. The Alzheimer's Disease Neuroimaging Initiative (ADNI): MRI methods. J. Magn. Reson. Imaging 27, 685-91 (2008).

38. Smith, S. M. et al. Advances in functional and structural MR image analysis and implementation as FSL. Neuroimage 23, S208-S219 (2004).

39. Jenkinson, M., Beckmann, C. F., Behrens, T. E. J., Woolrich, M. W. \& Smith, S. M. Fsl. Neuroimage 62, 782-90 (2012).

40. Pruim, R. H. R. et al. ICA-AROMA: A robust ICA-based strategy for removing motion artifacts from fMRI data. Neuroimage 112, 267-277 (2015).

41. Pruim, R. H. R., Mennes, M., Buitelaar, J. K. \& Beckmann, C. F. Evaluation of ICA-AROMA and alternative strategies for motion artifact removal in resting state fMRI. Neuroimage 112, 278-287 (2015).

42. Birn, R. M., Diamond, J. B., Smith, Ma \& Bandettini, P. a. Separating respiratory-variation-related fluctuations from neuronalactivity-related fluctuations in fMRI. Neuroimage 31, 1536-48 (2006).

43. Harvey, A. K. et al. Brainstem functional magnetic resonance imaging: disentangling signal from physiological noise. J. Magn. Reson. Imaging 28, 1337-44 (2008).

44. Diukova, A. et al. Separating neural and vascular effects of caffeine using simultaneous EEG-FMRI: differential effects of caffeine on cognitive and sensorimotor brain responses. Neuroimage 62, 239-49 (2012).

45. Brooks, J. C. W. et al. Physiological noise modelling for spinal functional magnetic resonance imaging studies. Neuroimage 39, 680-692 (2008).

\section{Acknowledgements}

The authors would like to thank Mark Tanner and Jonathan Howard for their invaluable help and expertise in the early stages of this study, and would also like to extend thanks to the Imanova Centre for Imaging Sciences for general support of the work.

\section{Author Contributions}

M.B.W., A.M., and R.D.N. conceived and designed the experiments, M.B.W., A.M., G.L., and R.D.N. conducted the data collection, M.B.W., A.M., G.L., O.S.K., L.D., and R.D.N. contributed to aspects of the data analysis, M.B.W. and R.D.N. wrote an initial draft of the manuscript, and all authors contributed edits to the final version.

Additional Information

Supplementary information accompanies this paper at doi:10.1038/s41598-017-11872-z

Competing Interests: The authors declare that they have no competing interests.

Publisher's note: Springer Nature remains neutral with regard to jurisdictional claims in published maps and institutional affiliations.

Open Access This article is licensed under a Creative Commons Attribution 4.0 International License, which permits use, sharing, adaptation, distribution and reproduction in any medium or format, as long as you give appropriate credit to the original author(s) and the source, provide a link to the Creative Commons license, and indicate if changes were made. The images or other third party material in this article are included in the article's Creative Commons license, unless indicated otherwise in a credit line to the material. If material is not included in the article's Creative Commons license and your intended use is not permitted by statutory regulation or exceeds the permitted use, you will need to obtain permission directly from the copyright holder. To view a copy of this license, visit http://creativecommons.org/licenses/by/4.0/.

(C) The Author(s) 2017 